\title{
Exploring an ultracold Fermi-Fermi mixture: Interspecies Feshbach resonances and scattering properties of ${ }^{6} \mathrm{Li}$ and ${ }^{40} \mathrm{~K}$
}

\author{
E. Wille, ${ }^{1,2}$ F.M. Spiegelhalder, ${ }^{1}$ G. Kerner, ${ }^{1}$ D. Naik, ${ }^{1}$ A. Trenkwalder,${ }^{1}$ G. Hendl, ${ }^{1}$ F. Schreck,,${ }^{1}$ \\ R. Grimm,${ }^{1,2}$ T.G. Tiecke,${ }^{3}$ J.T.M. Walraven,${ }^{3}$ S.J.J.M.F. Kokkelmans,${ }^{4}$ E. Tiesinga,${ }^{5}$ and P.S. Julienne ${ }^{5}$ \\ ${ }^{1}$ Institut für Quantenoptik und Quanteninformation, \\ Österreichische Akademie der Wissenschaften, 6020 Innsbruck, Austria \\ ${ }^{2}$ Institut für Experimentalphysik und Forschungszentrum für Quantenphysik, Universität Innsbruck, 6020 Innsbruck, Austria \\ ${ }^{3}$ Van der Waals-Zeeman Institute of the University of Amsterdam, 1018 XE, The Netherlands \\ ${ }^{4}$ Eindhoven University of Technology, P.O. Box 513, 5600 MB Eindhoven, The Netherlands \\ ${ }^{5}$ Joint Quantum Institute, National Institute of Standards and Technology \\ and University of Maryland, Gaithersburg, Maryland 20899-8423, USA
}

(Dated: October 30, 2018)

\begin{abstract}
We report on the observation of Feshbach resonances in an ultracold mixture of two fermionic species, ${ }^{6} \mathrm{Li}$ and ${ }^{40} \mathrm{~K}$. The experimental data are interpreted using a simple asymptotic bound state model and full coupled channels calculations. This unambiguously assigns the observed resonances in terms of various $s$ - and $p$-wave molecular states and fully characterizes the ground-state scattering properties in any combination of spin states.
\end{abstract}

PACS numbers: 34.50.-s, 67.85.-d, 05.30.Fk

Fermion pairing and Fermi superfluidity are key phenomena in superconductors, liquid ${ }^{3} \mathrm{He}$, and other fermionic many-body systems. Our understanding of the underlying mechanisms is far from being complete, in particular for technologically relevant high- $\mathrm{T}_{\mathrm{c}}$ superconductors. The emerging field of ultracold atomic Fermi gases has opened up unprecedented possibilities to realize versatile and well-defined model systems. The control of interactions, offered in a unique way by Feshbach resonances in ultracold gases, is a particularly important feature. Such resonances have been used to achieve the formation of bosonic molecules in Fermi gases and to control pairing in many-body regimes [1, 2, 3, 4, 5].

So far all experiments on strongly interacting Fermi systems have been based on two-component spin mixtures of the same fermionic species, either ${ }^{6} \mathrm{Li}$ or ${ }^{40} \mathrm{~K}$ [1, 2]. Control of pairing is achieved via a magnetically tunable $s$-wave interaction between the two states. After a series of experiments on balanced spin mixtures with equal populations of the two states, recent experiments on ${ }^{6} \mathrm{Li}$ have introduced spin imbalance as a new degree of freedom and begun to explore novel superfluid phases [6, 7]. Mixing two different fermionic species leads to unprecedented versatility and control. Unequal masses and the different responses to external fields lead to a large parameter space for experiments and promise a great variety of new phenomena $[8,9,10,11,12$. The combination of the two fermionic alkali species, ${ }^{6} \mathrm{Li}$ and ${ }^{40} \mathrm{~K}$, is a prime candidate to realize strongly interacting FermiFermi systems.

In this Letter, we realize a mixture of ${ }^{6} \mathrm{Li}$ and ${ }^{40} \mathrm{~K}$ and identify heteronuclear Feshbach resonances [14, 15, 16]. This allows us to characterize the basic interaction properties. Figure 11 shows the atomic ground-state energy structure. We label the energy levels $\mathrm{Li}|i\rangle$ and

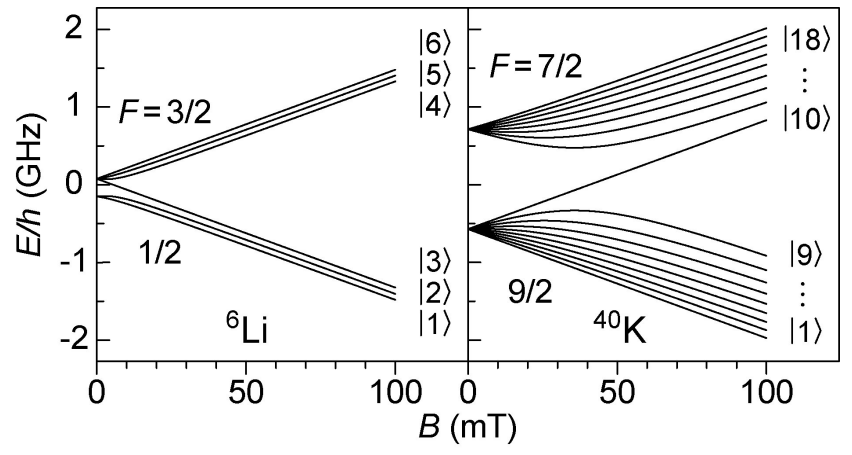

Figure 1: Ground state energies of ${ }^{6} \mathrm{Li}$ and ${ }^{40} \mathrm{~K}$ versus magnetic field.

$\mathrm{K}|j\rangle$, counting the states with rising energy. The hyperfine splitting of ${ }^{6} \mathrm{Li}$ is $(3 / 2) a_{\mathrm{hf}}^{\mathrm{Li}} / h=228.2 \mathrm{MHz}$. For ${ }^{40} \mathrm{~K}$, the hyperfine structure is inverted and the splitting amounts to $(9 / 2) a_{\mathrm{hf}}^{\mathrm{K}} / h=-1285.8 \mathrm{MHz}$ [17. For the low-lying states with $i \leq 3$ and $j \leq 10$, the projection quantum numbers are given by $m_{\mathrm{Li}}=-i+3 / 2$ and $m_{\mathrm{K}}=j-11 / 2$. A $\mathrm{Li}|i\rangle \mathrm{K}|j\rangle$ mixture can undergo rapid decay via spin relaxation if exoergic two-body processes exist that preserve the total projection quantum number $M_{\mathrm{F}}=m_{\mathrm{Li}}+m_{\mathrm{K}}=-i+j-4$. Whenever one of the species is in the absolute ground state and the other one is in a low-lying state $(i=1$ and $j \leq 10$ or $j=1$ and $i \leq 3)$, spin relaxation is strongly suppressed [18].

We prepare the mixture in an optical dipole trap, which is formed by two $70 \mathrm{~W}$-laser beams (wavelength $1070 \mathrm{~nm}$ ), crossing at an angle of $12^{\circ}$ [19]. The dipole trap is loaded with about $10^{7}{ }^{6} \mathrm{Li}$ atoms and a few $10^{4}{ }^{40} \mathrm{~K}$ atoms from a two-species magneto-optical trap (MOT). At this stage the trap depth for ${ }^{6} \mathrm{Li}\left({ }^{40} \mathrm{~K}\right)$ is $1.7 \mathrm{mK}(3.6 \mathrm{mK})$ and the trap oscillation frequencies are 


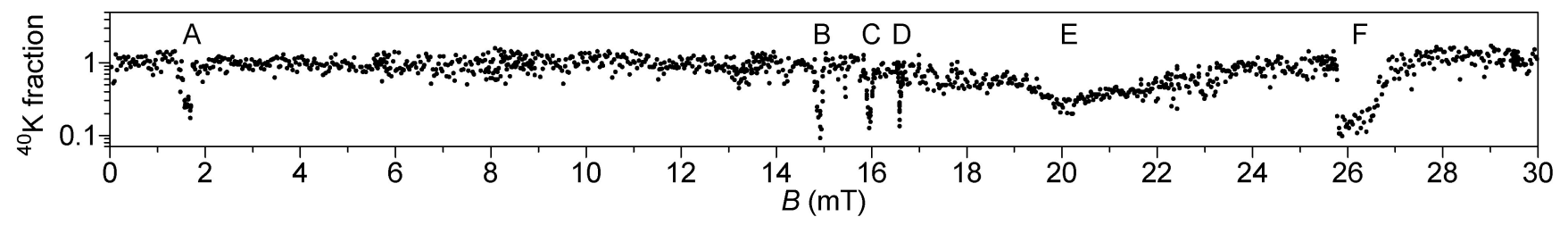

Figure 2: Feshbach scan of the $\mathrm{Li}|1\rangle \mathrm{K}|2\rangle$ mixture. The remaining fraction of ${ }^{40} \mathrm{~K}$ atoms relative to off-resonant regions after $10 \mathrm{~s}$ interaction with ${ }^{6} \mathrm{Li}$ atoms is shown as a function of magnetic field. Loss features A, B, C, D, and F are due to interspecies Feshbach resonances. Loss feature $\mathrm{E}$ is caused by a ${ }^{40} \mathrm{~K} p$-wave Feshbach resonance [13].

$13 \mathrm{kHz}(7.3 \mathrm{kHz})$ and $1.7 \mathrm{kHz}(1.0 \mathrm{kHz})$ in radial and axial directions. After preparation of the internal states of the atoms [19, a balanced mixture of $\mathrm{Li}|1\rangle$ and $\mathrm{Li}|2\rangle$ atoms together with $\mathrm{K}|1\rangle$ atoms is obtained. We perform evaporative cooling at a magnetic field of $76 \mathrm{mT}$ close to the $83.4 \mathrm{mT}$ Feshbach resonance between $\mathrm{Li}|1\rangle$ and Li $|2\rangle$ [1, 2, by reducing the optical dipole trap depth exponentially by a factor of 70 over $2.5 \mathrm{~s}$. We observe that potassium remains thermalized with lithium during the evaporation. This results in $10^{5} \mathrm{Li}|1\rangle$ and $10^{5}$ $\mathrm{Li}|2\rangle$ atoms together with $10^{4} \mathrm{~K}|1\rangle$ atoms at a temperature of $4 \mu \mathrm{K}$. This three-component Fermi mixture serves as a starting point to prepare several different stable two-component mixtures, namely $\mathrm{Li}|2\rangle \mathrm{K}|1\rangle, \mathrm{Li}|1\rangle \mathrm{K}|1\rangle$, $\mathrm{Li}|1\rangle \mathrm{K}|2\rangle$, or $\mathrm{Li}|1\rangle \mathrm{K}|3\rangle$ with $M_{\mathrm{F}}=-5,-4,-3,-2$, respectively. Atoms in the $\mathrm{K}|1\rangle$ state are transferred to the desired state with adiabatic radio-frequency sweeps. Population in unwanted states is pushed out of the trap by pulses of resonant light [19]. Finally, to increase the collision rate, the sample is compressed by increasing the power of the optical trap. The temperature rises to $12 \mu \mathrm{K}$ and the peak density of lithium (potassium) increases to about $10^{12} \mathrm{~cm}^{-3}$ (few $10^{11} \mathrm{~cm}^{-3}$ ).

We detect Feshbach resonances by observing enhanced atom loss at specific values of the magnetic field [3], which is caused by three-body decay. For each mixture we perform a magnetic field scan with a resolution of $0.03 \mathrm{mT}$ between 0 and $74 \mathrm{mT}$ ( 0 to $40 \mathrm{mT}$ for the $\mathrm{Li}|1\rangle \mathrm{K}|3\rangle$ mixture). A scan consists of many experimental cycles, each with a total duration of about one minute during which the mixture is submitted for ten seconds to a specific magnetic field value. The quantity of remaining atoms is measured by recapturing the atoms into the MOTs and recording their fluorescence light.

In Fig. 2, we show a loss spectrum of $\mathrm{Li}|1\rangle \mathrm{K}|2\rangle$. A striking feature is that the potassium atom number decreases by an order of magnitude at specific values of the magnetic field. Since the mixture contains an order of magnitude more lithium than potassium atoms, the lithium atom number does not change significantly by interspecies inelastic processes. Therefore, the potassium loss is exponential and near complete. In order to distinguish loss mechanisms involving only one species from those involving two species, we perform additional loss measurements, using samples of either pure ${ }^{6} \mathrm{Li}$ or pure
${ }^{40} \mathrm{~K}$. Loss features A, B, C, D, and F only appear using a two-species mixture. Loss feature $\mathrm{E}$ persists in a pure ${ }^{40} \mathrm{~K}$ sample and can be attributed to a potassium $p$-wave Feshbach resonance [13]. On the basis of the experimental data only, we can not unambiguously attribute loss feature $\mathrm{C}$ to an interspecies Feshbach resonance, since it coincides with a known ${ }^{6} \mathrm{Li} p$-wave resonance [20, 21].

Our main findings on positions and widths $\Delta B$ of

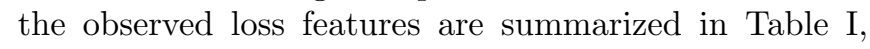
together with the results of two theoretical models described in the following.

Our analysis of the data requires finding the solutions for the Hamiltonian $H=H_{\alpha}^{\mathrm{hf}}+H_{\beta}^{\mathrm{hf}}+H^{\mathrm{rel}}$. To underline the generality of our model, we refer to $\mathrm{Li}$ as $\alpha$ and to $\mathrm{K}$ as $\beta$. The first two terms represent the hyperfine and Zeeman energies of each atom, $H^{\mathrm{hf}}=$ $\left(a_{\mathrm{hf}} / \hbar^{2}\right) \mathbf{s} \cdot \mathbf{i}+\gamma_{e} \mathbf{s} \cdot \mathbf{B}-\gamma_{n} \mathbf{i} \cdot \mathbf{B}$, where $\mathbf{s}$ and $\mathbf{i}$ are the single-atom electron and nuclear spin, respectively, and $\gamma_{e}$ and $\gamma_{n}$ are the respective gyromagnetic ratios. The Hamiltonian of relative motion is

$$
H^{\mathrm{rel}}=\frac{\hbar^{2}}{2 \mu}\left(-\frac{d^{2}}{d r^{2}}+\frac{l(l+1)}{r^{2}}\right)+\sum_{S=0,1} V_{S}(r) P_{S},
$$

where $\mu$ is the reduced mass, $r$ is the interatomic separation, and $l$ is the angular momentum quantum number for the relative motion. Defining the total electron spin as $\mathbf{S}=\mathbf{s}_{\alpha}+\mathbf{s}_{\beta}$, the projection operator $P_{S}$ either projects onto the $S=0$ singlet or $S=1$ triplet spin states. The potential $V_{S}(r)$ is thus either for the singlet $\mathrm{X}^{1} \Sigma$ or triplet $\mathrm{a}^{3} \Sigma$ state. This Hamiltonian $H$ conserves both $l$ and $M_{\mathrm{F}}$.

Our first method to locate the Feshbach resonances is inspired by a two-body bound state model for homonuclear [22] and heteronuclear [14] systems. We have expanded this previous work to include the part of the hyperfine interaction that mixes singlet and triplet levels. This mixing is crucial for the present analysis. We refer to this model as the Asymptotic Bound state Model (ABM).

The ABM model expands the bound state solutions $\left|\Psi^{l}\right\rangle$ for each $l$ in terms of $\left|\psi_{S}^{l}\right\rangle\left|S, M_{S}, \mu_{\alpha}, \mu_{\beta}\right\rangle$ where $\left|\psi_{S}^{l}\right\rangle$ is the asymptotic last bound eigenstate of the potential $V_{S}(r)+\hbar^{2} l(l+1) /\left(2 \mu r^{2}\right)$ and $\left|S, M_{S}, \mu_{\alpha}, \mu_{\beta}\right\rangle$ are spin functions where $M_{S}, \mu_{\alpha}$ and $\mu_{\beta}$ are the magnetic quantum numbers of $\mathbf{S}, \mathbf{i}_{\alpha}$ and $\mathbf{i}_{\beta}$, respectively. Only spin 
Table I: Feshbach resonances in collisions between ${ }^{6} \mathrm{Li}$ and ${ }^{40} \mathrm{~K}$ in a range from 0 to $76 \mathrm{mT}$. For their positions $B_{0}$, we give the center of the measured loss features and the results from both the ABM and coupled channels calculations. The first columns give the ${ }^{6} \mathrm{Li}$ and ${ }^{40} \mathrm{~K}$ channel indices $i$ and $j$ and the projection quantum number $M_{\mathrm{F}}=-i+j-4$. Note that the experimental width of a loss feature, $\Delta B$, is not the same thing as the width $\Delta B_{\mathrm{s}}$ related to the scattering length singularity. The latter is only defined for $s$-wave resonances, and not for the observed $p$-wave resonances. The typical statistical and systematic error in the experimental $B_{0}$ is about $0.05 \mathrm{mT}$ for $s$-wave resonances.

\begin{tabular}{|c|c|c|c|c|c|c|}
\hline \multirow[b]{2}{*}{$i, j$} & \multirow[b]{2}{*}{$M_{\mathrm{F}}$} & \multicolumn{2}{|c|}{ Experiment } & \multirow{2}{*}{$\begin{array}{c}\mathrm{ABM} \\
B_{0} \\
(\mathrm{mT})\end{array}$} & \multicolumn{2}{|c|}{ Coupled channels } \\
\hline & & $\begin{array}{c}B_{0} \\
(\mathrm{mT})\end{array}$ & $\begin{array}{c}\Delta B \\
(\mathrm{mT})\end{array}$ & & $\begin{array}{c}B_{0} \\
(\mathrm{mT})\end{array}$ & $\begin{array}{c}\Delta B_{\mathrm{s}} \\
(\mathrm{mT})\end{array}$ \\
\hline 2,1 & -5 & $21.56^{a}$ & 0.17 & 21.67 & 21.56 & 0.025 \\
\hline 1,1 & -4 & 15.76 & 0.17 & 15.84 & 15.82 & 0.015 \\
\hline 1,1 & -4 & 16.82 & 0.12 & 16.92 & 16.82 & 0.010 \\
\hline 1,1 & -4 & 24.9 & 1.1 & 24.43 & 24.95 & $p$ wave \\
\hline 1,2 & -3 & 1.61 & 0.38 & 1.39 & 1.05 & $p$ wave \\
\hline 1,2 & -3 & 14.92 & 0.12 & 14.97 & 15.02 & 0.028 \\
\hline 1,2 & -3 & $15.95^{a}$ & 0.17 & 15.95 & 15.96 & 0.045 \\
\hline 1,2 & -3 & 16.59 & 0.06 & 16.68 & 16.59 & 0.0001 \\
\hline 1,2 & -3 & 26.3 & 1.1 & 26.07 & 26.20 & $p$ wave \\
\hline 1,3 & -2 & not ol & erved & 1.75 & 1.35 & $p$ wave \\
\hline 1,3 & -2 & 14.17 & 0.14 & 14.25 & 14.30 & 0.036 \\
\hline 1,3 & -2 & 15.49 & 0.20 & 15.46 & 15.51 & 0.081 \\
\hline 1,3 & -2 & 16.27 & 0.17 & 16.33 & 16.29 & 0.060 \\
\hline 1,3 & -2 & 27.1 & 1.4 & 27.40 & 27.15 & $p$ wave \\
\hline
\end{tabular}

${ }^{a}$ Near coincidences with lithium $p$-wave resonances [20] 21].

functions with the same conserved $M_{\mathrm{F}}=M_{S}+\mu_{\alpha}+\mu_{\beta}$ are allowed. Note that $S, M_{S}, \mu_{\alpha}, \mu_{\beta}$ are good quantum numbers for large magnetic field. Expanding $\left|\Psi^{l}\right\rangle$ in this basis and assuming that the overlap $\left\langle\psi_{0}^{l} \mid \psi_{1}^{l}\right\rangle$ is unity [23], the coupled bound state energies are found by diagonalizing the interaction matrix [19].

The energies $E_{S}^{l}$ of the last bound state of the $S=0$ and 1 potentials are eigenvalues of Eq. (1), and serve as free parameters in the ABM model. We can reduce this to only two binding energy parameters $E_{0}=-E_{0}^{0}$ and $E_{1}=-E_{1}^{0}$ if we use information about the actual shape of the potential. We can do this using model potentials derived from Refs. 24 and 25], and the van der Waals coefficient $C_{6}=2322 E_{\mathrm{h}} a_{0}^{6}\left(E_{\mathrm{h}}=4.35974 \times 10^{-18} \mathrm{~J}\right.$ and $a_{0}=0.0529177 \mathrm{~nm}$ ) [26]. Each $E_{S}$ can be varied by making small changes to the short range potential while keeping $C_{6}$ fixed. The energy $E_{S}$ uniquely determines both the $s$-wave scattering length as well as $E_{S}^{l}$ for $l>0$.

Figure 3 shows the bound state energies of the ABM model as a function of magnetic field for $M_{\mathrm{F}}=-3$. Feshbach resonances occur at the crossings of bound states and threshold. We find a good fit for the experimental resonance positions for parameters $E_{0} / h=716(15) \mathrm{MHz}$ and $E_{1} / h=425(5) \mathrm{MHz}$, where the uncertainty represents one standard deviation, see Table I.

For additional analysis we have also used exact, yet

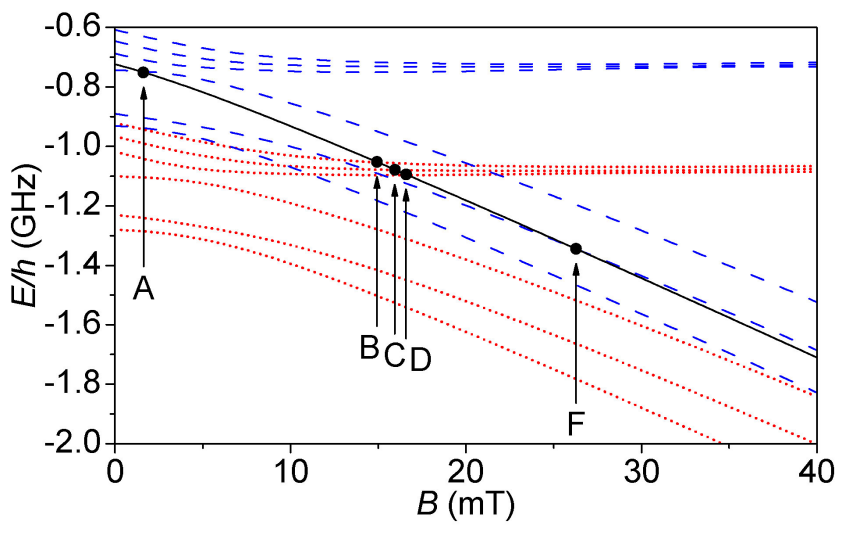

Figure 3: Bound state energies versus magnetic field. Dotted (dashed) lines indicate the $s$-wave ( $p$-wave) states. The twobody threshold for the $\mathrm{Li}|1\rangle \mathrm{K}|2\rangle$ collision channel $\left(M_{\mathrm{F}}=-3\right)$ is indicated by the solid line. The dots and the corresponding arrows indicate the measured resonance positions (see Fig. 2).

much more computationally complex coupled channels calculations [27, varying the short range potential as discussed above. An optimized fit to the measured resonance positions gives $E_{0} / h=721(10) \mathrm{MHz}$ and $E_{1} / h=$ 426(3) MHz. This corresponds to a singlet scattering length of $52.1(3) a_{0}$ and a triplet scattering length of 63.5(1) $a_{0}$. Thus, within the fitting accuracy to the experimental data, the prediction of the ABM model agrees with the result of the full coupled channels calculation. Table I shows the coupled channels resonance locations and widths for a representative calculation with $E_{0} / h=$ $720.76 \mathrm{MHz}$ and $E_{1} / h=427.44 \mathrm{MHz}$. The $s$-wave resonance width $\Delta B_{\mathrm{s}}$ is defined by $a_{\mathrm{s}}(B)=a_{\mathrm{bg}}\left(1-\Delta B_{\mathrm{s}} /(B-\right.$ $\left.B_{0}\right)$ ), where $a_{\mathrm{bg}}$ is the background scattering length near the resonance position $B_{0}$. Note that $\Delta B_{\mathrm{s}}$ need not be the same as the empirical width $\Delta B$ of a loss-feature. All resonances except the $M_{\mathrm{F}}=-3 p$-wave resonance near $1.6 \mathrm{mT}$ agree with the measured positions within $0.13 \mathrm{mT}$. Fine-tuning of the long range potential would be needed to fit this resonance to comparable accuracy. Figure 4 shows the calculated $s$-wave scattering lengths and $p$-wave elastic cross sections versus magnetic field $B$ for this model. The background scattering length $a_{\mathrm{bg}}$ for the $s$-wave resonances is approximately $63 \mathrm{a}_{0}$.

The accuracy and computational simplicity of the ABM model make resonance assignments very efficient, allowing rapid feedback between the experiment and theory during the exploratory search for resonances. As the ABM model in its present form does not yield the width of the resonances, the prediction of a resonance position is not expected to be more accurate than the corresponding experimental resonance width. For the ${ }^{6} \mathrm{Li}^{4}{ }^{40} \mathrm{~K}$ mixture, the ABM model predicts hundreds of further resonances in various $s$ - and $p$-wave channels up to $0.1 \mathrm{~T}[19$.

A remarkable feature of the ${ }^{6} \mathrm{Li}^{4}{ }^{40} \mathrm{~K}$ system is the large widths of the $p$-wave resonances near $25 \mathrm{mT}$, which by 


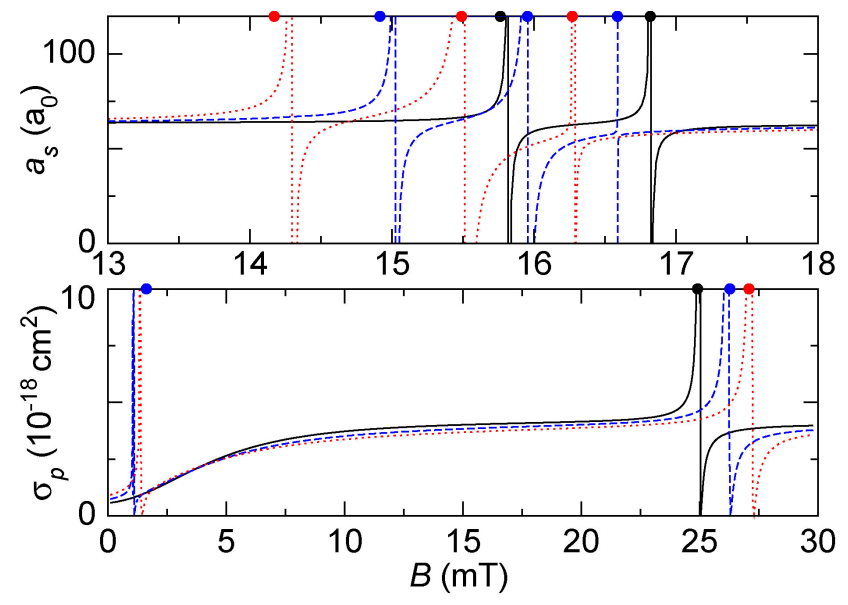

Figure 4: Results from coupled channels calculations for the magnetic-field dependence of the $s$-wave scattering length $a_{s}(B)$ (upper panel) and the $m_{l}=0$ contribution to the $p$ wave elastic scattering cross section $\sigma_{p}(E)$ for $E / k_{B}=12 \mu \mathrm{K}$ (lower panel) for the channels in Table-1 with $M_{\mathrm{F}}=-4$ (solid line), -3 (dashed line), and -2 (dotted line). The dots indicate the measured resonance locations.

far exceeds the width of the observed $s$-wave resonances. Naively, one would expect the $s$-wave resonances to be wider than their $p$-wave counterparts because of the different threshold behavior. However, in the present case the difference in magnetic moments between the atomic threshold and the relevant molecular state is found to be anomalously small, which stretches out the thermally broadened $p$-wave resonance features over an unusually wide magnetic field range. Also the asymmetry of the loss feature supports its interpretation as a $p$-wave resonance [20, 21, 28].

An important issue for future experiments is the character of the $s$-wave resonances, i.e. the question whether they are entrance-channel or closed-channel dominated [3, 4]. All our observed resonances are rather narrow and thus closed-channel dominated. The existence of entrance-channel dominated resonances would be of great interest to experimentally explore BEC-BCS crossover physics [1, 2] in mixed Fermi systems. However, our coupled channels calculations for a partial set of predicted resonances have not yet found any such resonances, and their existence seems unlikely in view of the moderate values of the background scattering lengths [3, 4].

In conclusion, we have characterized the interaction properties in an ultracold mixture of ${ }^{6} \mathrm{Li}$ and ${ }^{40} \mathrm{~K}$ atoms by means of Feshbach spectroscopy and two theoretical models. The results are of fundamental importance for all further experiments in the emerging field of FermiFermi mixtures. Further steps will be the formation of bosonic ${ }^{6} \mathrm{Li}^{40} \mathrm{~K}$ molecules through a Feshbach resonance and evaporative cooling towards the creation of a heteronuclear molecular Bose-Einstein condensate.

A double-degenerate mixture of ${ }^{6} \mathrm{Li}$ and ${ }^{40} \mathrm{~K}$ was re- cently demonstrated in a magnetic trap [29].

We thank E. Tiemann for stimulating discussions. The Innsbruck team acknowledges support by the Austrian Science Fund (FWF) and the European Science Foundation (ESF) within the EuroQUAM project. TGT and JTMW acknowledge support by the FOM-Program for Quantum gases. SJJMFK acknowledges support from the Netherlands Organization for Scientific Research (NWO). PSJ acknowledges partial support by the U.S. Office of Naval Research.

[1] M. Inguscio, W. Ketterle, and C. Salomon, eds., Ultracold Fermi Gases (IOS Press, Amsterdam, 2007), Proceedings of the International School of Physics "Enrico Fermi", Course CLXIV, Varenna, 20-30 June 2006.

[2] S. Giorgini, L. P. Pitaevskii, and S. Stringari, arXiv:0706.3360

[3] C. Chin, R. Grimm, P. Julienne, and E. Tiesinga, in preparation for Rev. Mod. Phys.

[4] T. Köhler, K. Góral, and P. S. Julienne, Rev. Mod. Phys. 78, 1311 (2006).

[5] I. Bloch, J. Dalibard, and W. Zwerger, arXiv:0704.3011.

[6] M. W. Zwierlein, A. Schirotzek, C. H. Schunck, and W. Ketterle, Science 311, 492 (2006).

[7] G. B. Partridge et al., Science 311, 503 (2006).

[8] W. V. Liu and F. Wilczek, Phys. Rev. Lett. 90, 047002 (2003).

[9] D. S. Petrov, C. Salomon, and G. V. Shlyapnikov, J. Phys. B: At. Mol. Opt. Phys. 38, S645 (2005).

[10] M. Iskin and C. A. R. Sá de Melo, Phys. Rev. Lett. 97, 100404 (2006).

[11] G. Orso, L. P. Pitaevskii, and S. Stringari, arXiv:0709.1690

[12] D. S. Petrov et al., Phys. Rev. Lett. 99, 130407 (2007).

[13] C. A. Regal, C. Ticknor, J. L. Bohn, and D. S. Jin, Phys. Rev. Lett. 90, 053201 (2003).

[14] C. A. Stan et al., Phys. Rev. Lett. 93, 143001 (2004).

[15] S. Inouye et al., Phys. Rev. Lett. 93, 183201 (2004).

[16] F. Ferlaino et al., Phys. Rev. A 73, 040702 (2006); ibid. 74, 039903(E) (2006).

[17] E. Arimondo, M. Inguscio, and P. Violino, Rev. Mod. Phys. 49, 31 (1977).

[18] A. Simoni et al., Phys. Rev. Lett. 90, 163202 (2003).

[19] More details will be published elsewhere.

[20] J. Zhang et al., Phys. Rev. A 70, 030702(R) (2004).

[21] C. H. Schunck et al., Phys. Rev. A 71, 045601 (2005).

[22] A. J. Moerdijk, B. J. Verhaar, and A. Axelsson, Phys. Rev. A 51, 4852 (1995).

[23] The actual values are 0.979 for $\mathrm{l}=0$ and 0.965 for $\mathrm{l}=1$.

[24] H. Salami et al., J. Chem. Phys. 126, 194313 (2007).

[25] M. Aymar and O. Dulieu, J. Chem. Phys. 122, 204302 (2005).

[26] A. Derevianko, J. F. Babb, and A. Dalgarno, Phys. Rev. A 63, 052704 (2001).

[27] H. T. C. Stoof, J. M. V. A. Koelman, and B. J. Verhaar, Phys. Rev. B 38, 4688 (1988).

[28] F. Chevy et al., Phys. Rev. A 71, 062710 (2005).

[29] M. Taglieber et al., Phys. Rev. Lett. 100, 010401 (2008). 